\title{
Finding the Goldilocks zone for chemical crystallography via Laue single-crystal neutron diffraction - what have we learned from KOALA to improve KOALA 2.0?
}

\author{
A.J. Edwards, R.O. Piltz \\ Australian Centre for Neutron Scattering, Australian Nuclear Science and Technology Organization, \\ New Illawarra Rd, Lucas Heights, N.S.W., Australia
}

Alison.Edwards@ansto.gov.au

KOALA is a single-crystal Laue neutron diffractometer standing at the end of guide position of the supermirror guide TG3 at the OPAL reactor, ANSTO. The instrument was initially modelled closely on VIVALDI[1], an instrument available in the user program at the ILL from 2001-2010. The elegantly simple concept of the instrument employs a cylindrical neutron sensitised image plate detector which is used to record a series of diffraction images from a suitable number of crystal positions to provide a sufficient data set from which valid model parameters can be derived to answer questions regarding material properties which cannot be adequately derived from more readily available methods, most particularly X-ray diffraction and more recently the hybrid methodology of quantum crystallography.

Our initial practice with the instrument adhered largely to that shared with us by the scientists at the ILL. This early experience[2] was the commencement of a steep learning curve which has, with a very limited number of other instruments brought single-crystal neutron diffraction into greater use in chemistry and chemical crystallography in the second decade of the 21 st century. Key developments have been (i) the installation of an Oxford Cryosystems COBRA ${ }^{\mathrm{TM}}$ nitrogen cryostream which facilitates handling of oxygen and moisture sensitive compounds (which encompass a significant fraction of the proposals received for the instrument) and (ii) the development of a user accessible data reduction for the diffraction images. From the first proposal round for the instrument in 2009 exciting chemistry was proposed for experiments which exceeded the nominal maximum primitive unit cell volume for the recording of useful diffraction images. A simple work around for this has been to reduce the resolution of the images by manipulation of the temperature at which they are recorded - in order to obtain data against which a model may be refined.

More commonly though, it is observed that crystals for which the unit cell volume is relatively large tend, where they can be grown to a size sufficient for Laue neutron diffraction, to have a mosaic spread which limits the resolution of the pattern observed without manipulating the temperature to further reduce the resolution.

With careful attention to experimental detail and the availability of discretionary beam-time access it has been possible to undertake studies of important new materials in timeframes which have resulted in the publication of the single-crystal neutron diffraction study with the chemistry it underpins, rather than as a stand alone paper reporting only the neutron study result. It is of particular importance to note that in the case of hydride containing compounds, it can be critical to prove the location of the hydride via neutron diffraction and even a low resolution study can provide the necessary proof. In consequence of their publication with the chemistry, papers from KOALA are now submitted to and published in journals of the highest standing [4-7].

Having achieved a more routine applicability of neutron diffraction in chemical crystallography, we reached a point where elctronic components of KOALA had exceeded their serviceable lifespans and contemplation of replacing this aspect of the instrument led us to realise that reworking the existing mechanical elements with new electronics posed significant challenges and would cost a large fraction of the potential cost of building a new instrument. We are fortunate that the decision was reached to design a new instrument which is allowing us to optimise key design elements to yield maximum flexibility of the instrument across all of its possible applications in chemistry, physics, materials science and crystallography. The instrument is currently under construction and should be available for users in the second half of 2022.

[1] C Wilkinson, JA Cowan, DAA Myles, F Cipriani, GJ Mclntyre, (2002), Neutron News, 13, 37-41.

[2] AJ Edwards, (2011) Australian Journal of Chemistry 64, 869-872

[3] RO Piltz,(2018) Journal of Applied Crystallography 51, 635-645 and 963-965

[4] M Garçon, C Bakewell, GA Sackman, AJP White, RI Cooper, AJ Edwards, MR Crimmin (2019) Nature 574, 390-393

[5] SJ Bonyhady, D Collis, N Holzmann, AJ Edwards, RO Piltz, G Frenking, A Stasch C Jones, (2018) Nature Comms 9 , 3079

[6] JAB Abdalla, A Caise, CP Sindlinger, R Tirfoin, AL Thompson, AJ Edwards, S Aldridge, (2017) Nature chemistry 9, 1256-1262

[7] R Chen, G Qin, S Li, AJ Edwards, RO Piltz, I Del Rosal, L Maron, D Cui, J Cheng Angewandte Chemie 132, 11346-11351

Keywords: neutron diffraction; Laue diffraction; chemical crystallography; instrumentation; structure

Acta Cryst. (2021), A77, C382 